Abstract P44 Table 1 Clinical, demographic and MRI indicators in hospitalised and stable COPD patients and controls

\begin{tabular}{|c|c|c|c|c|c|}
\hline & Hospitalised COPD & Stable COPD & Controls & $P$ value & Post hoc tests \\
\hline Number (n) & 23 & 17 & 23 & - & - \\
\hline Age (years) ${ }^{1}$ & $69 \pm 11$ & $67 \pm 7$ & $66 \pm 7$ & 0.477 & - \\
\hline Gender $(\% \text { male })^{2}$ & $61 \%$ & $60 \%$ & $46 \%$ & 0.540 & - \\
\hline $\mathrm{FEV}_{1}(\% \text { predicted })^{3}$ & $51 \pm 18$ & $54 \pm 24$ & - & 0.604 & - \\
\hline \multirow[t]{2}{*}{ Smoking (pack years) ${ }^{4}$} & \multirow[t]{2}{*}{$43(35)$} & \multirow[t]{2}{*}{$60(25)$} & \multirow[t]{2}{*}{$0(3)$} & \multirow[t]{2}{*}{$<0.0001$} & $\mathrm{H}>\mathrm{C}^{* *}$ \\
\hline & & & & & $\mathrm{S}>\mathrm{C}^{* *}$ \\
\hline Cognitive function $(/ 30)^{4}$ & $23.0(5)$ & $27.0(2)$ & $28.5(3)$ & $<0.0001$ & $\mathrm{H}>\mathrm{C}^{* *}$ \\
\hline White matter lesion volume $(\mathrm{ml})^{5}$ & $8.9(17.0)$ & $7.4(16.5)$ & $3.0(7.2)$ & 0.026 & - \\
\hline Normalised whole brain volume $(\mathrm{ml})^{5}$ & $1399(113)$ & $1424(121)$ & $1381(111)$ & 0.736 & - \\
\hline \multirow[t]{2}{*}{ Normalised brain ventricular volume $(\mathrm{ml})^{6}$} & \multirow[t]{2}{*}{$71.1 \pm 27.6$} & \multirow[t]{2}{*}{$52.8 \pm 22.8$} & \multirow[t]{2}{*}{$53.1 \pm 19.1$} & \multirow[t]{2}{*}{0.016} & $\mathrm{H}>\mathrm{S}^{*}$ \\
\hline & & & & & $\mathrm{H}>\mathrm{C}^{*}$ \\
\hline
\end{tabular}

Values are given as mean \pm standard deviation or median (interquartile range). Statistical tests: 1 . One-way analysis of variance; 2 . Pearson Chi-square test; 3 . Independent $t$ test; 4 . Kruskal-Wallis test with post hoc Dunn-Bonferroni; 5 . Kruskal-Wallis test; 6 . One-way analysis of variance with post hoc Bonferroni; ${ }^{*} p<0.05 ;{ }^{* *} p<0.0001$.

within the last year (stable) and 23 healthy age-matched controls, were recruited. Exclusion criteria included neurological or psychiatric disorders, cerebrovascular disease or hypertension. Cognition was measured using the Montreal Cognitive Assessment. T1-weighted (T1W), diffusion tensor (DTI) and Fluid Attenuated Inversion Recovery (FLAIR) were acquired using a Philips 3T scanner. The following brain indicators were calculated: normalised whole brain and ventricular volumes (scan: T1W, software: SIENAX), white matter microstructural fractional anisotropy and mean diffusivity - compared voxelwise using tract-based spatial statistics (DTI, FSL), and white matter lesion volume (FLAIR, dispunc). Ethical approval was obtained (15/LO/0425).

Results Cognition was clinically impaired in hospitalised COPD (median (IQR); 23.0 (5)) but not in stable patients $(27.0$ (2)) or controls (28.5 (3)), with a significant difference only between hospitalised patients and controls $(p<0.0001)$. No significant differences were found in normalised whole brain volume, however, normalised brain ventricular volume was significantly greater in hospitalised COPD, compared to stable COPD $(p=0.046)$ and controls $(p=0.028)$. Hospitalised and stable COPD patients had widespread significant reductions in fractional anisotropy and increased mean diffusivity throughout the white matter skeleton, compared to controls $(p<0.001)$. Hospitalised patients had the highest white matter lesion volume, however this was not significantly different between groups.

Conclusions Hospitalised COPD patients have greater cognitive impairment compared to stable COPD and controls with evidence of greater ventricular and white matter lesion volumes and damaged white matter microstructure. Mechanisms behind these neuropathological processes and possible links to observed cognitive dysfunction remain unclear, but may involve ischaemic smallvessel disease.

\section{P45 NON-INVASIVE METHODS FOR THE ESTIMATION OF MPAP IN COPD PATIENTS USING CARDIAC MRI}

${ }^{1} \mathrm{CS}$ Johns, ${ }^{1} \mathrm{D}$ Capener, ${ }^{1} \mathrm{C}$ Hammerton, ${ }^{1} \mathrm{~K}$ Shotton, ${ }^{2} \mathrm{DG}$ Kiely, ${ }^{1} \mathrm{JM}$ Wild, ${ }^{1} \mathrm{AJ}$ Swift. ${ }^{1}$ The University of Sheffield, Sheffield, UK; ${ }^{2}$ Sheffield Teaching Hospitals, Sheffield, UK
Background Pulmonary hypertension (PH) is important in COPD as it predicts death and hospitalisation. The diagnosis is made by right heart catheter (RHC). Several predictive cardiac MRI (CMR) models have been proposed to estimate mean pulmonary artery pressure (mPAP):

1. VMI/IVS: $-4.6+$ (interventricular septal angle (IVS) $\mathrm{x} 0.23$ ) $+(\text { ventricular mass index }(\mathrm{VMI}) \times 16.3)^{1}$

2. PA/RV: $\times 21.806+(\mathrm{IVS} \times 0.31)+(\mathrm{VMI} \times 11.5)+$ $($ Diastolic pulmonary artery $(\mathrm{PA})$ area $\times 0.01)-(\mathrm{PA}$ relative area change $\times 0.22$ )

3. Alpha-index: minimum PA area/right ventricular ejection fraction $^{2}$

The predictive value of these models in a COPD population with suspected $\mathrm{PH}$ remains unknown, so we aimed to assess their diagnostic accuracy.

Methods All consecutive patients referred to a $\mathrm{PH}$ centre from April 2012 to October 2015 with suspected PH were assessed. Any patient with a formal diagnosis of COPD was included. Sensitivity and specificity were calculated using diagnostic cut-offs published in the literature (VMI/IVS $=32, \mathrm{PA} / \mathrm{RV}$ model $=25$, alpha index $=7.2$ ). Ethical approval was granted.

Results 1864 patients were referred to the PH centre, 145 had a documented diagnosis of COPD, 102 had MRI and RHC within 90 days. All CMR models showed good correlation with RHC measured mPAP (Pearson's R for (i) VMI/IVS $=0.689$, (ii) PA/RV model $=0.732$ and (iii) Alpha-index $=0.527$ ). Sensitivity and specificity for (i) VMI/IVS were $92 \%$ and $79 \%$, (ii) PA/RV model $80 \%$ and $93 \%$ and (iii) for Alpha index $100 \%$ and $13 \%$ respectively. An ROC curve for the diagnosis of $\mathrm{PH}$ for each of the models is provided.

Conclusion VMI/IVS and PA/RV models both have good accuracy in the detection of PH in COPD patients. Alpha-index had a low specificity, largely due to a low diagnostic threshold. As such these models are useful in the assessment of $\mathrm{PH}$, and likely prognosis in patients with COPD. 


\section{ROC comparing all CMRI MPAP predictive models for making a diagnosis of $\mathrm{PH}$}

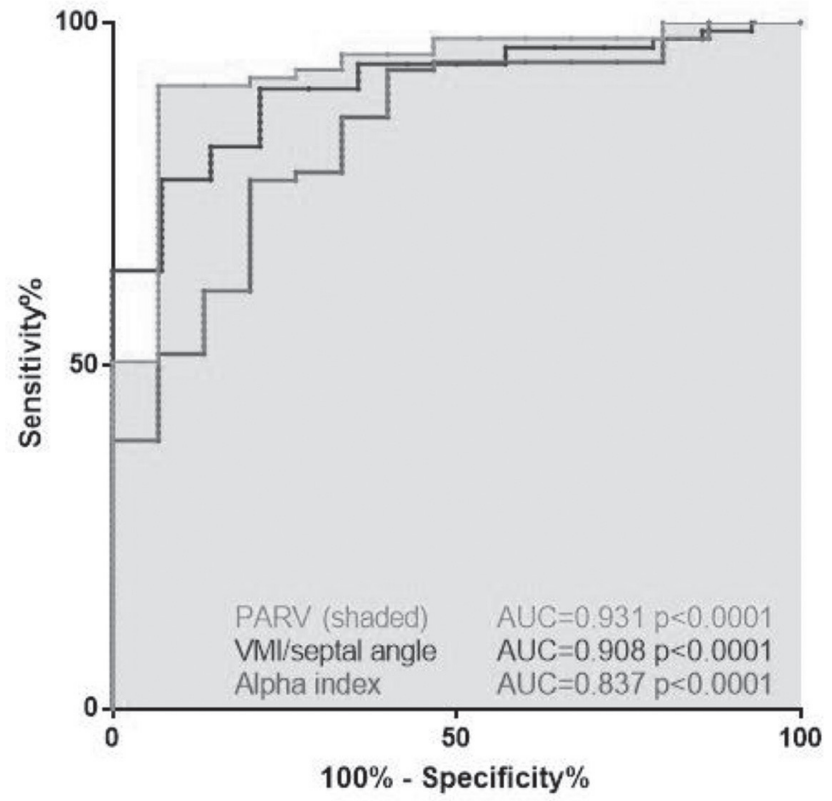

Abstract P45 Figure 1 ROC comparing all CMRI mPAP predictive models for making a diagnosis of $\mathrm{PH}$

\section{REFERENCES}

1 Swift AJ, et al. Noninvasive estimation of PA Pressure, Flow, and Resistance With CMR Imaging. JACC Cardiovasc Imaging 2013;6(10):1036-47.

2 Moral $\mathrm{S}$, et al. New index alpha improves detection of pulmonary hypertension in comparison with other cardiac magnetic resonance indices. Int J Cardio 2012;161(1):25-30.

\section{P46 ASSESSMENT OF AORTIC STIFFNESS AND CORRELATION WITH LUNG FUNCTION IN PATIENTS WITH COPD USING CARDIAC MAGNETIC RESONANCE}

${ }^{1} E$ De Garate, ${ }^{1} G$ Biglino, ${ }^{1} A$ Wilson, ${ }^{2} E$ Baker, ${ }^{2} \mathrm{P}$ Jones, ${ }^{1} \mathrm{C}$ Bucciarelli-Ducci, ${ }^{3} \mathrm{~J}$ Dodd. ${ }^{1}$ NIHR Bristol Cardiovascular Biomedical Research Unit, Bristol Heart Institute, Bristol, UKi ${ }^{2}$ Division of Clinical Sciences St. George's University of London, London, UK; ${ }^{3}$ Academic Respiratory Unit University of Bristol, Southmead Hospital, Bristol, UK

\subsection{6/thoraxjnl-2016-209333.189}

Introduction: COPD has been associated with increased cardiovascular risk, although the mechanisms for this are still unclear. Proposed theories include increased systemic inflammation and accelerated ageing resulting in arterial stiffness. We aimed to evaluate aortic distensibility using cardiac MRI in patients with COPD compared to an age-matched non COPD, 'healthy' smoker control group.

Methods we recruited 49 subjects, of which 27 had diagnosis of COPD and FEV1/FVC < 70\%; and 21 age-matched normal smoker controls (mean age 64 years \pm 10 ). We acquired data including age, gender, smoking status, number of packs of cigarettes per year, and FEV1/FVC ratio. MRI images were acquired using a 3.0T scanner, and analysed using CVI42 software. Left ventricle and right ventricle function and volumes were evaluated using short axis SSFP cine. Aortic distensibility was measured using a validated method that takes in consideration aortic maximal and minimal areas from axial SSFP cine acquired perpendicular to the vessel.
Results Aortic distensibility was reduced in the COPD patients compared to control $\left(0.0022610 \times 10^{-3} \mathrm{~mm} \mathrm{Hg}^{-1}\right.$ vs $\left.0.004337 \times 10^{-3} \mathrm{~mm} \mathrm{Hg}^{-1}, \mathrm{p}=0.003\right)$. The distensibility of descending aorta was similar in both groups $(p=0.06)$. Ejection fraction and biventricular volumes were also similar in the two groups. Univariant analysis demonstrated a significant relationship between ascending aorta distensibility and FEV1/FVC ratio. There was no difference when comparing distensibility with smoking status or number of packs per year. Linear regression demonstrated that the degree of aortic distensibility was directly proportional to FEV1/FVC ratio

Conclusion Patients with COPD have significantly increased aortic stiffness measured by cardiac magnetic resonance. This was observed in the presence of normal LV/RV systolic function in both groups. This difference was related to FEV1/FVC, and was independent of smoking. Preserved FEV1/FVC showed more elastic ascending aortas. Reduced aortic distensibility could represent the early phase changes in cardiovascular function but further research is needed.

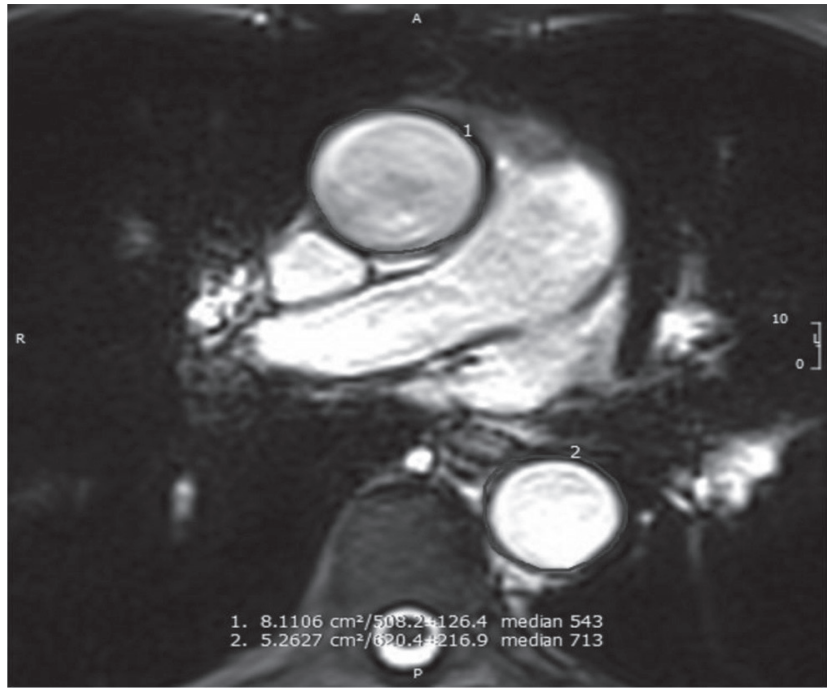

Abstract P46 Figure 1 Contouring of ascending and descending aortic area on axial SSFP cine

\section{\begin{tabular}{l|l} 
P47 & THE INFLUENCE OF MUSCLE MASS IN THE
\end{tabular} ASSESSMENT OF LOWER LIMB STRENGTH IN COPD}

${ }^{1} \mathrm{R}$ Trethewey, ${ }^{1} \mathrm{D}$ Esliger, ${ }^{1} \mathrm{E}$ Petherick, ${ }^{2} \mathrm{R}$ Evans, ${ }^{3} \mathrm{~N}$ Greening, ${ }^{4} \mathrm{~B}$ James, ${ }^{1} \mathrm{~A}$ Kingsnorth, ${ }^{2} \mathrm{M}$ Morgan, ${ }^{1} \mathrm{M}$ Orme, ${ }^{2} \mathrm{~S}$ Singh, ${ }^{3} \mathrm{~L}$ Sherar, ${ }^{2} \mathrm{~N}$ Toms, ${ }^{2} \mathrm{M}$ Steiner. ${ }^{1} \mathrm{NCSEM}$, Loughborough University, Loughborough, UK: ${ }^{2}$ Respiratory Biomedical Research Unit, Glenfield Hospital, Leicester, UK; ${ }^{3}$ Institute of Lung Health, Leicester, UK; ${ }^{4}$ Leicester University, Leicester, UK

\subsection{6/thoraxjnl-2016-209333.190}

Introduction and objectives Lower limb muscle strength measured by Quadriceps Maximal Voluntary Contraction (QMVC) provides valuable functional and prognostic information in people with COPD. Reference equations providing normal values for QMVC have been reported, some requiring measurement of muscle mass. It is unclear whether including muscle mass in the calculation significantly alters predicted values in COPD. We addressed this question by deriving reference equations for QMVC with and without the inclusion of whole body assessment of muscle mass in a cohort of healthy volunteers and 